\title{
On the orbits of the outer satellites of Jupiter
}

\author{
T. Yokoyama ${ }^{1}$, M. T. Santos ${ }^{1}$, G. Cardin ${ }^{1}$, and O. C. Winter ${ }^{2}$ \\ 1 Universidade Estadual Paulista, IGCE - DEMAC Caixa Postal 178, CEP 13.500-970 Rio Claro (São Paulo), Brasil \\ 2 Universidade Estadual Paulista, FEG, Grupo de Dinâmica Orbital e Planetologia da UNESP, Caixa Postal 205, \\ CEP 12.516-410 Guaratinguetá (São Paulo), Brasil \\ e-mail: ocwinter@feg.unesp.br
}

Received 11 September 2002 / Accepted 31 January 2003

\begin{abstract}
In this work we study the basic aspects concerning the stability of the outer satellites of Jupiter. Including the effects of the four giant planets and the Sun we study a large grid of initial conditions. Some important regions where satellites cannot survive are found. Basically these regions are due to Kozai and other resonances. We give an analytical explanation for the libration of the pericenters $\varpi-\varpi_{J}$. Another different center is also found. The period and amplitude of these librations are quite sensitive to initial conditions, so that precise observational data are needed for Pasiphae and Sinope. The effect of Jupiter's mass variation is briefly presented. This effect can be responsible for satellite capture and also for locking $\varpi-\varpi_{J}$ in temporary libration.
\end{abstract}

Key words. planets and satellites: general - solar system: general

\section{Introduction}

The recent discovery of outer and irregular satellites of Uranus and Saturn (Gladman et al. 1998, 2000, 2001) confirms the remarkable similarity in the architecture of the giant planets. All of them have a system of rings (and sometimes small satellites) which are close to the planet. Next, they have a region populated by a system of large satellites, whose orbits are in general very regular, i.e., almost circular and planar. Finally, at a very large distance, a few small satellites, with high inclination and large eccentricity complete the scenario around the giant planets. In this work we focus our attention on these distant and irregular satellites. They are strongly disturbed by the Sun and their dynamics are far from being clean keplerian orbits such as are found at small distances of the planet. Due to their large distance from the host planet, not only the Sun, but the remaining giant planets can exert non-negligible perturbations. Moreover, since the ratio of the semi major axes $a / a^{\prime}$ is large, in general, analytical treatment by perturbation methods is not efficient. In particular, in the case of the Outer Retrograde Satellites (ORS) of Jupiter: Carme, Ananke, Sinope, Pasiphae, these features are crucial. Among the well known satellites in the solar System, probably they have the largest $a / a^{\prime}$, and show remarkable variations in eccentricity and inclination. However in spite of these variations, their orbits seem to be very stable and if some chaos exists, only Sinope is weakly affected (Saha \& Tremaine 1993).

Send offprint requests to: T. Yokoyama,

e-mail: tadashi@ms.rc.unesp.br
Within the last two years a significant number of irregular Jupiter satellites has been discovered (Sheppard \& Jewitt 2002). So far these authors discovered 21 new satellites and they estimate that there are about 100 irregular satellites which could be classified in 3 different dynamical groups. Since the orbital elements of these satellites are still very new, in this work we consider mainly the two well known groups: prograde or direct group (Leda, Lysithea, Himalaia, Elara) and retrograde group (Carme, Ananke, Sinope, Pasiphae).

Probably Hénon made the first detailed study of the stability of the retrograde satellites of Jupiter. He reduced the general restricted three body problem to Hill's case and in a long series of papers, he presented an impressive discussion of the main features of the solutions (Hénon 1965a,b, 1966a,b, 1969, 1970a,b). Hill's case is a simplified version of the restricted three body problem, where the inclination is neglected and Jupiter's orbit is circular. In spite of these simplifications, some remarkable results are obtained. Perhaps the most important concerns the stability of the retrograde satellites: all four outermost satellites are well inside a significant stable region. More precisely, Hénon shows that the family of simple retrograde periodic orbits is always linearly stable even when the distance from the planet reaches very high values (Hénon 1970a,b). The existence of these stable regions, even in the restricted three body problem is very important, since they are directly related to the capture problem. It is believed that due to the action of some additional forces (such as gas drag, mass variation, etc.) a temporary capture can be converted into a permanent one. But for this phenomenon to occur, a non-negligible stable region 
should exist, since in general, during the capture evolution, all these orbits suffer considerable variations in eccentricity and inclination. In this way, Huang \& Innanen (1993) made a numerical study of the stability and the capture regions for retrograde jovicentric satellites in the restricted three-body problem. All the initial conditions satisfied the mirror theorem (at initial instant, the satellite is in conjunction with Jupiter and the Sun and its velocity is perpendicular to the direction Sun-Jupiter). Typical integration time was about 20000 years.

In this work we investigate a large region of the phase space including the outer satellites of Jupiter. In our numerical simulation we consider the four giant planets defining a large grid of initial conditions in the $a \times I$ plane ( $a=$ semi major axis, $I=$ inclination). We show that there are important regions where strong instabilities preclude the existence of satellites. Some of these instabilities are due to the Kozai resonance, while others seem to be related to the interaction of different resonances. Our typical integration time was about 650000 to one million years. Due to the number of initial conditions to be integrated, we decided to shorten the integration time in order to explore a larger grid.

The dynamics of retrograde satellites of Jupiter, in particular Pasiphae, was also studied by Whipple \& Shelus (1993). The typical integration time was about 100000 years, and all the planets were included, while the total mass of the Galilean satellites was added to that of Jupiter. They discovered a possible $\varpi-\varpi_{J}$ secular resonance which locks Pasiphae's longitude of perijove and Jupiter's longitude of perihelion. According to these authors this locking could be a mechanism that protects Pasiphae from strong solar perturbation. For the period of libration of this resonance, Whipple \& Shelus found 13500 years and for the amplitude $60^{\circ}$. In an interesting paper Saha \& Tremaine (1993) revisited the problem of outer satellites, taking an integration over an interval of $2 \mathrm{Myr}$. This time, the initial conditions were different from those used by the previous authors and in fact, their filtered values show libration for Pasiphae. They also mention a similar temporary libration for Sinope. In view of these secular resonances, Saha \& Tremaine suggest that this locking mechanism could constrain theories of the formation of the retrograde satellites. According to our numerical integrations the values found by Whipple \& Shelus are very sensitive to the initial conditions, so that accurate observations seem to be decisive for these satellites.

To explain the theoretical possibility of a locking mechanism between the apse of the planet and the satellite, we investigate this resonance starting from a simple model derived from the averaged system of the restricted three body problem. It is shown that the location of the equilibrium point of this libration depends on the constant $H+G$ which is a first integral in the simplified model. Indeed, one more center of libration (in opposition to the previous one) is found; however, its region of stability seems to be very small. We also consider a few examples including the case in which Jupiter's mass varies according to the model presented in Fleming \& Hamilton (2001). Temporary capture in $\varpi-\varpi_{\odot}$ occurs very often during the mass variation.

This paper is organized in the following way: Sect. 1 is devoted to the derivation of averaged systems. In spite of the limitations of the averaged systems we show that some qualitative information can be gained with these simplified models. In Sects. 2 and 3 a theoretical model showing the possibility of the libration of $\varpi-\varpi_{\odot}$ is obtained from the averaged system. It is shown that in the simplified model, this resonance is the same as the well-known one in the classical asteroidal problem. Also the main effects due to the Kozai resonance are discussed. In Sect. 4 we show the results of the numerical integration of a large grid of initial conditions. Some significant regions where the eccentricity attains prohibitive values are shown on a graylevel scale plotted in the $a \times I$ plane. Finally, in Sect. 5 we briefly show some results for the libration of $\varpi-\varpi_{\odot}$ under the influence of Jupiter's mass variation.

\section{The averaged system}

In a coordinate system centered on the host planet (Jupiter), the basic equations of a satellite disturbed by only one disturber (the Sun, for instance) are:

$\frac{\mathrm{d}^{2} \boldsymbol{r}}{\mathrm{d} t^{2}}=-\frac{k^{2}(M+m)}{r^{3}} \boldsymbol{r}-k^{2} M_{\odot}\left(\frac{\boldsymbol{r}-\boldsymbol{r}_{\odot}}{\left|r-r_{\odot}\right|^{3}}+\frac{\boldsymbol{r}_{\odot}}{r_{\odot}^{3}}\right)$.

The meaning of the variables is:

- $M, M_{\odot}$ : mass of the planet and of the Sun, respectively;

- $m$ : mass of the satellite;

- $\boldsymbol{r}, \boldsymbol{r}_{\odot}$ : position vector of the satellite and of the Sun;

$-k^{2}$ : gravitation constant.

In the above equation, the expression factored by $M_{\odot}$ defines the classical disturbing function $R^{*}$ :

$R^{*}=k^{2} M_{\odot}\left(\frac{1}{\Delta}-\frac{r \cdot r_{\odot}}{r_{\odot}^{3}}\right)$

where $\Delta$ is the distance between the satellite and the Sun.

When the ratio of the semi major axes $\rho=a / a_{\odot}$ is small, $R^{*}$ can be expanded into a power series of this quantity so that analytic perturbative solutions are constructed for the elements. For the ORS, $\rho$ is not small, about 0.03 . However, from a qualitative point of view, it is very instructive to review some basic aspects of this kind of expansion. For example, in the EarthMoon problem, $\rho$ is about 0.0026 . For this value, the convergence of the series is still slow (Brouwer \& Clemence 1961). Even so, Delaunay $(1860,1867)$ successfully derived sophisticated perturbation methods obtaining a very powerful theory for the lunar problem. For Caliban, the ratio $\rho=a / a_{\odot}$ is very similar $(\approx 0.0025)$. The difference is that Caliban's inclination and eccentricity are not small: $\approx\left(139^{\circ}-143^{\circ}\right)$ and $\approx(0.1-0.3)$. However, once $R^{*}$ is expanded into powers of $a / a_{\odot}$, we can show that an averaged system can be derived in closed form for arbitrary eccentricities and inclinations. Therefore, in this case, the averaged solutions should be able to describe qualitatively the long-term variation of the motion, as they do in the case of small eccentricities and inclinations, since no truncation is done during the averaging procedure. To analyze this question briefly, let us take a simple model as given in (2) where we consider a massless satellite, the Sun and a planet. The disturbing 

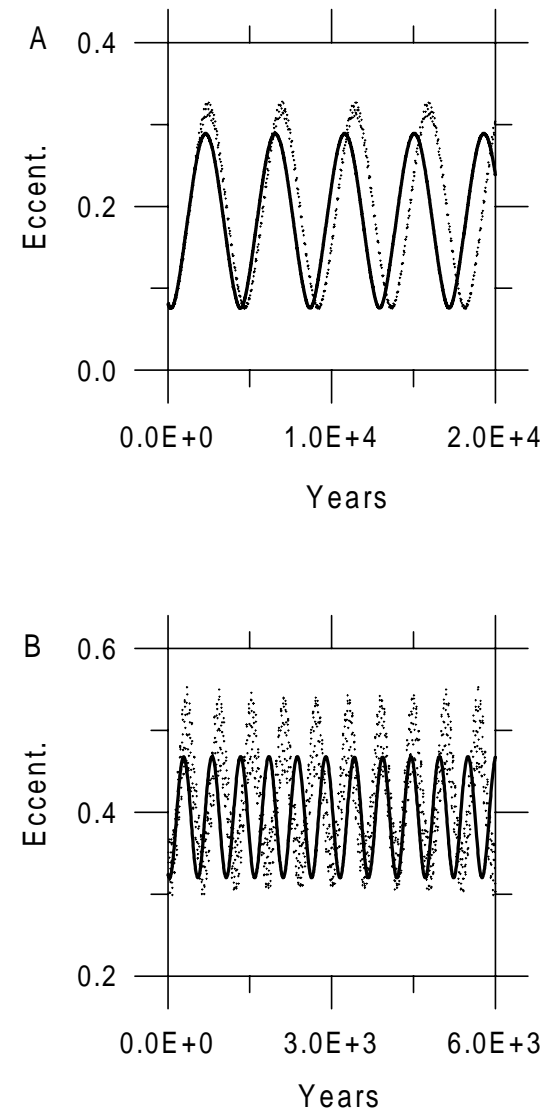

Fig. 1. Time variation of the eccentricity for Caliban A) and Prospero B). The dotted curve is obtained from direct integration while the thick curve comes from the averaged system. Initial conditions: Caliban: $(a=0.047081, e=0.0807123, w=339.07160, \Omega=174.99098$, $I=139.68829)$, Prospero: $(a=0.1093574, e=0.3240699, w=$ 170.56106, $\Omega=320.08612, I=146.31924)$.

function $R^{\star}$ truncated at the fourth order is:

$$
\begin{aligned}
R^{*}= & \frac{k^{2} M_{\odot}}{r_{\odot}}\left\{\frac{r^{2}}{r_{\odot}^{2}}\left(-\frac{1}{2}+\frac{3}{2} \cos ^{2} S\right)+\frac{r^{3}}{r_{\odot}^{3}}\left(-\frac{3}{2} \cos S+\frac{5}{2} \cos ^{3} S\right)\right. \\
& \left.+\frac{r^{4}}{r_{\odot}^{4}}\left(\frac{3}{8}-\frac{30}{8} \cos ^{2} S+\frac{35}{8} \cos ^{4} S\right)\right\}
\end{aligned}
$$

where $S$ is the angle between the vector direction of the satellite and of the Sun. Therefore:

$$
\begin{aligned}
\cos S= & \frac{1}{4}(1+\cos I)\left(1+\cos I_{\odot}\right) \\
& \times \cos \left(f+w+\Omega-f_{\odot}-w_{\odot}-\Omega_{\odot}\right) \\
& +\frac{1}{4}(1+\cos I)\left(1-\cos I_{\odot}\right) \\
& \times \cos \left(f+w+\Omega+f_{\odot}+w_{\odot}-\Omega_{\odot}\right) \\
& +\frac{1}{4}(1-\cos I)\left(1+\cos I_{\odot}\right) \\
& \times \cos \left(f+w-\Omega+f_{\odot}+w_{\odot}+\Omega_{\odot}\right) \\
& +\frac{1}{4}(1-\cos I)\left(1-\cos I_{\odot}\right) \\
& \times \cos \left(f+w-\Omega-f_{\odot}-w_{\odot}+\Omega_{\odot}\right) \\
& +\frac{1}{2} \sin I \sin I_{\odot}\left(\cos \left(f+w-f_{\odot}-w_{\odot}\right)\right. \\
& \left.-\cos \left(f+w+f_{\odot}+w_{\odot}\right)\right)
\end{aligned}
$$

where the elements of the satellite are:

- $f=$ true anomaly

$-w=$ argument of the pericenter

$-\Omega=$ longitude of the node

$-I=$ inclination

and the similar variables with $\odot$ refer to the Sun.

Since we are interested in the long-term variation, a double average of $R^{*}$ with respect to the mean anomalies $l$ and $l_{\odot}$ is applied in order to eliminate short period variations.

$R=\frac{1}{4 \pi^{2}} \int_{0}^{2 \pi} \int_{0}^{2 \pi} R^{*} \mathrm{~d} l \mathrm{~d} l_{\odot}$.

Taking some classical relations of the Keplerian problem (Brouwer \& Clemence 1961) we can perform the above double integration in closed form without any expansion in eccentricity or inclination. Moreover, choosing for instance the ecliptic 2000 as the reference plane, it is possible to introduce directly any secular planetary theory for the long-term motion of Jupiter (Laskar 1988). However, usually the final analytic expression of the averaged $R$ in Eq. (5) (even for third order in $\rho$ ) increases enormously and the computation time for the integration of the averaged equations, (even considering only one disturber) is very significant.

To overcome in part this inconvenience and also to avoid expansion in the ratio $a / a_{\odot}$ we can use the Schubart extended method (Moons 1994).

Figure 1 shows the decisive role played by the ratio $a / a_{\odot}$. At the top we have the variation of Caliban's eccentricity obtained from Schubart's method (thick curve) and from direct integration (dotted curve), respectively. Here the ratio of the semi-major axes is $\approx 0.00244$, similar to the Earth-Moon case. In spite of a small phase difference in time, the agreement is acceptable for this value of $\rho$. At the bottom we have the corresponding curves for Prospero whose $\rho$ is twice the previous $(\approx 0.00566)$. We want to emphasize that in the Schubart method, in general very significant variations, whose amplitudes are almost equal to or even greater than those due to long period terms, are eliminated even though no expansion in $\rho$, eccentricity, or inclination is used. This is clearly seen for Prospero's eccentricity. The reason is that for the present $\rho$ direct numerical integrations clearly show large variations that occur in a very short time interval (less than the orbital period of Uranus). However, these large variations are completely eliminated because the averaging method removes any oscillation whose period is shorter than Uranus' orbital period, no matter its amplitude. In Fig. 1 some improvement is still possible by choosing properly the initial mean conditions for the averaged system, however the main problem still remains. Actually, the situation is more dramatic in the case of large $\rho$ or eccentricity, since close approaches are the main source of significant and abrupt oscillations that occur in a short interval of time. Therefore whenever $\rho$ is large (like in ORS), none of these fast variation, no matter their amplitude, appear in the averaged system.

For the present problem and from a quantitative point of view, expansions in $\rho$ are not adequate; however, the basic 
structure of the phase space which is due to the long period should be preserved in the averaged system, at least qualitatively. This is the point we want to explore next. Since Schubart's method does not provide an analytic expression for the disturbing function, we consider expanded forms as given in Eq. (3).

\section{Libration of the apses and the Kozai resonance}

Let us return to the problem of the libration of the difference of the longitudes of the apses, $\varpi-\varpi_{J}$, mentioned in the previous Section. According to Whipple \& Shelus (1993), an analytic treatment of the solution is required to establish rigorously the existence of the locking mechanism between Pasiphae's apsis and Jupiter's apsis.

A tentative method to clarify this question is given in Saha $\&$ Tremaine (1993). They used a semi-numerical approach for the case of Sinope-Jupiter. Here we derive an alternative and analytic answer, taking advantage of the averaged system in its simplest form. Actually a twofold development plan is presented here, because we also explain some important features of the phase space due to the Kozai resonance.

To consider the averaged disturbing function $R$ (Eq. (5)) in its simplest case, let us take the orbit of the planet as the reference plane, that is $I_{\odot}=0$ in Eq. (4). Since we are interested in the $\varpi-\varpi_{\odot}$ resonant terms, we have to consider at least terms of order $\rho^{3}$ in the disturbing function (Eq. (3)). For the present calculations we included terms of $\operatorname{order} \rho^{4}$, and we also tested fifth order terms. However since fifth order terms are also factored by $e_{\odot}$ they are negligibly small.

Following some classical calculations and defining:

$$
\begin{aligned}
b_{1}= & -5 / 2 e-15 / 8 e^{3} \\
b_{2}= & -35 / 8 e^{3} \\
c_{1}= & k^{2} a^{2} \frac{1}{a_{\odot}^{3}\left(1-e_{\odot}^{2}\right)^{\frac{3}{2}}} \\
c_{2}= & k^{2} a^{3} \frac{e_{\odot}}{a_{\odot}^{4}\left(1-e_{\odot}^{2}\right)^{\frac{5}{2}}} \\
c_{3}= & k^{2} a^{4} \frac{1}{a_{\odot}^{5}\left(1-e_{\odot}^{2}\right)^{\frac{7}{2}}} \\
d_{1}= & 1+5 e^{2}+15 / 8 e^{4} \\
d_{2}= & \frac{21}{8} e^{2}\left(2+e^{2}\right) \\
d_{3}= & \frac{63}{8} e^{4} \\
c I= & \cos I \\
s I= & \sin I \\
R_{2}= & \frac{1}{8}\left(1+3 / 2 e^{2}\right)\left(3 c I^{2}-1\right)+\frac{15}{16} e^{2} s I^{2} \cos 2 w \\
R_{3}= & \frac{1}{64}\left\{\left(-3+33 c I+15 c I^{2}-45 c I^{3}\right) b_{1} \cos \left(\varpi_{\odot}-\Omega+w\right)\right. \\
& +\left(-3-33 c I+15 c I^{2}+45 c I^{3}\right) b_{1} \cos \left(\varpi_{\odot}-\Omega-w\right) \\
& +\left(15-15 c I-15 c I^{2}+15 c I^{3}\right) b_{2} \cos \left(\varpi_{\odot}-\Omega+3 w\right) \\
& \left.+\left(15+15 c I-15 c I^{2}-15 c I^{3}\right) b_{2} \cos \left(\varpi_{\odot}-\Omega-3 w\right)\right\} \\
& \\
R^{2}-3 &
\end{aligned}
$$

$$
\begin{aligned}
R_{4}= & \frac{3}{16}\left(2+3 e_{\odot}^{2}\right) d_{1}-\frac{495}{1024} e_{\odot}^{2}-\frac{135}{256} c I^{2} \\
& -\frac{165}{512}+\frac{315}{512} c I^{4}+\frac{945}{1024} c I^{4} e_{\odot}^{2}-\frac{405}{512} c I^{2} e_{\odot}^{2} \\
& +\left(\frac{105}{512} c I^{4}+\frac{315}{1024} e_{\odot}^{2}-\frac{105}{256} c I^{2}+\frac{105}{512}\right. \\
& \left.-\frac{315}{512} c I^{2} e_{\odot}^{2}+\frac{315}{1024} c I^{4} e_{\odot}^{2}\right) d_{3} \cos (4 w) \\
& +\frac{105}{512}\left(c I^{3}-c I-\frac{1}{2} c I^{4}+\frac{1}{2}\right) d_{3} e_{\odot}^{2} \cos \left(4 w+2 \varpi_{\odot}-2 \Omega\right) \\
& +\frac{105}{512}\left(-c I^{3}+c I-\frac{1}{2} c I^{4}+\frac{1}{2}\right) d_{3} e_{\odot}^{2} \cos \left(4 w-2 \varpi_{\odot}+2 \Omega\right) \\
& +\left(\frac{45}{64} c I^{2}-\frac{45}{512}-\frac{315}{512} c I^{4}\right) e_{\odot}^{2} \cos \left(2 \varpi_{\odot}-2 \Omega\right) \\
& +\left(\frac{15}{16} c I^{2}+\frac{45}{32} c I^{2} e_{\odot}^{2}-\frac{45}{256} e_{\odot}^{2}-\frac{15}{128}\right. \\
& \left.-\frac{315}{256} c I^{4} e_{\odot}^{2}-\frac{105}{128} c I^{4}\right) d_{2} \cos (2 w) \\
+ & +\frac{15}{256}-\frac{45}{128} c I^{2}+\frac{75}{256} c I \\
& \left.+\frac{105}{256} c I^{4}-\frac{105}{256} c I^{3}\right) d_{2} e_{\odot}^{2} \cos \left(2 w+2 \varpi_{\odot}-2 \Omega\right) \\
+ & \left(\frac{15}{256}-\frac{45}{128} c I^{2}-\frac{75}{256} c I\right. \\
& \left.+\frac{105}{256} c I^{3}\right) d_{2} e_{\odot}^{2} \cos \left(2 w-2 \varpi_{\odot}+2 \Omega\right) \\
& \\
+ &
\end{aligned}
$$

Finally the averaged disturbing function truncated at order $\left(\frac{a}{a_{\odot}}\right)^{4}$ is given by:

$R=R_{2} c_{1}+R_{3} c_{2}+R_{4} c_{3}$.

We define $\varpi=\Omega+w$ for prograde orbits. For retrograde orbits, the definition of longitude of the pericenter should be slightly changed (Whipple \& Shelus 1993), that is, $\varpi=\Omega-w$. This new definition for $\varpi$ is necessary in order to satisfy classical D'Alembert properties in the expansion of the disturbing function (Nascimento \& Yokoyama 2003). With this definition the corresponding resonant Hamiltonian is obtained from the previous disturbing function (8) neglecting all terms in the periodic part which are not factored by $\cos (\Psi)$ or $\cos (2 \Psi)$ where $\Psi=\varpi-\varpi_{\odot}$.

$R=R_{2 r} c_{1}+R_{3 r} c_{2}+R_{4 r} c_{3}$

where

$$
\begin{aligned}
R_{2 r}= & \frac{1}{8}\left(1+3 / 2 e^{2}\right)\left(3 c I^{2}-1\right) \\
R_{3 r}= & \frac{1}{64}\left\{\left(-3+33 c I+15 c I^{2}-45 c I^{3}\right) b_{1} \cos (\Psi)\right. \\
R_{4 r}= & \frac{3}{16}\left(2+3 e_{\odot}^{2}\right) d_{1}-\frac{495}{1024} e_{\odot}^{2}-\frac{135}{256} c I^{2}-\frac{165}{512}+\frac{315}{512} c I^{4} \\
& +\frac{945}{1024} c I^{4} e_{\odot}^{2}-\frac{405}{512} c I^{2} e_{\odot}^{2}+\left(\frac{15}{256}-\frac{45}{128} c I^{2}+\frac{75}{256} c I\right. \\
& \left.+\frac{105}{256} c I^{4}-\frac{105}{256} c I^{3}\right) d_{2} e_{\odot}^{2} \cos (2 \Psi) .
\end{aligned}
$$


Let $G=n a^{2}\left(1-e^{2}\right)^{1 / 2}$ and $H=G \cos I$. Then in the canonical variables: $(\Psi,-G)$ the disturbing function (9) defines a Hamiltonian of one degree of freedom, where $H+G$ is an integral of motion. Then it is easy to draw the contours lines of the Hamiltonian. To fix the constant of motion, we chose the following values: $a=335.46107 R_{J}, e=0.411, I=140^{\circ}$, $a_{J}=10890.232 R_{J}, e_{J}=0.04785$ in Fig. $2 \mathrm{~A}$, while in the bottom (B): $a=335.46107 R_{J}, e=0.571741, I=143.49^{\circ}$ and $a_{J}=32670.696 R_{J}$. These figures remained almost unchanged with the inclusion of terms of order $\rho^{5}$.

Since the origin of our coordinates is fixed in the planet, the libration center is $\varpi-\varpi_{\odot}=0^{\circ}$, Fig. $2 \mathrm{~A}$.

In this figure the value of the eccentricity at the libration center is larger than those obtained by Whipple \& Shelus (1993), Saha \& Tremaine (1993). We believe that this difference is mainly due to the oversimplified model we use. In conclusion, we have shown that the libration of the apses, $\varpi-\varpi_{J}$, can be derived very easily with a simple model obtained from the averaged system. Therefore this libration is very similar to the well-known apsidal resonance in the planetary theory. According to this geometry, this configuration protects the satellites from closest approach to the Sun. The above result was obtained considering the restricted problem. Indeed a direct integration taking only Jupiter-Sun-satellite, shows a clear libration in the osculating elements, even for Sinope. Whether this libration remains if additional disturbers are included will be investigated in the next section.

In the Hamiltonian (9) the libration center moves to $\varpi-$ $\varpi_{\odot}=180^{\circ}$ if different values are chosen for the integral $H+G$. This is shown in Fig. 2B. To confirm this, we integrated the restricted three body problem taking the initial conditions mentioned before and also: $\Omega=110.15^{\circ}, w=109.02^{\circ}, l=27.38^{\circ}$, $\Omega_{J}=0^{\circ}, w_{J}=180^{\circ}, l_{J}=0^{\circ}$. In Fig. 3 the adopted semi major axis of the planet is fixed at three times its present value.

Recently Cuk et al. (2002) showed that, apparently, the Saturnian satellite $S / 2000 S 2$ is currently librating in $\varpi-\varpi_{\odot}=$ $180^{\circ}$ resonance. This is interesting because in this configuration the satellite is not in a protection mechanism like Pasiphae or Sinope.

Now let us investigate another important resonance which is related to the libration of $w$. To do that, we consider again a very simple model and write the disturbing function (8) in terms of the classical Delaunay variables:

$$
\begin{aligned}
& G=n a^{2}\left(1-e^{2}\right)^{\frac{1}{2}} \\
& H=G \cos I \\
& g=w \\
& h=\Omega .
\end{aligned}
$$

If we consider $e_{\odot}=0$ the Hamiltonian obtained from (8) is: $F=F(G, H, g)$, where $H$ is constant since the node is absent when $e_{\odot}=0$. Critical equilibrium points are given by:

$\frac{\mathrm{d} G}{\mathrm{~d} t}=\frac{\mathrm{d} w}{\mathrm{~d} t}=0$.

For $w=\pi / 2$ or $3 \pi / 2$ the first equation is immediately satisfied, so that $\frac{\mathrm{d} g}{\mathrm{~d} t}=0$ is easily solved, but it is clear that the solution
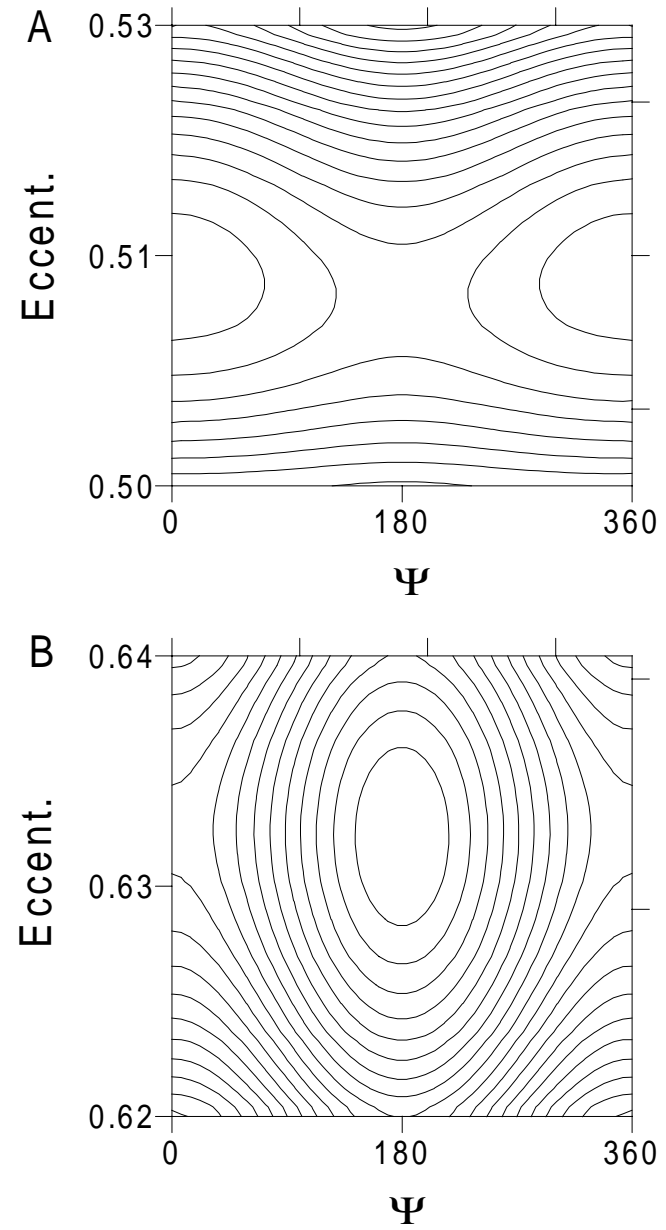

Fig. 2. Contours for the Hamiltonian (9) showing two possibilities of the libration of $\Psi=\varpi-\varpi_{\odot}$.

depends on the constant $H$. Actually if terms of order $\rho^{4}$ are neglected, we have:

$\frac{\mathrm{d} g}{\mathrm{~d} t}=\frac{c_{1}}{4 G^{3}}\left\{\frac{9 G^{4}}{L^{2}}-15 H^{2}\right\}$

therefore the equilibrium center can exist only if $\cos I^{2} \leq=0.6$, that is $I=\left[39^{\circ}, 140.8^{\circ}\right]$.

Since $F=F(G, H, g)$ is a one-degree-of-freedom problem, the trajectories in the $(e-w)$ plane are obtained from its contours (Fig. 4).

In Fig. 4A, the integral of motio $H$ was defined through: $a=320, e=0.001, I=110^{\circ}$, while at the bottom we used the same values, except $I=150^{\circ}$. This clearly shows that whenever I falls within the interval $I=\left[39.2^{0}, 140.8^{\circ}\right]$, the eccentricity can vary by a large amount, while outside this interval the variation is very small. Since $H$ is a constant, the variation of the inclination is always connected to variation of the eccentricity through $H=G \cos I=$ constant. Therefore in the case of the Kozai resonance, both eccentricity and inclination undergo large variations.

We emphasize that when only $O\left(\rho^{2}\right)$ terms are considered, $\Omega$ does not appear in the disturbing function, so that $H$ is a constant. Including higher-order terms, we still have a onedegree-of-freedom problem provided we consider $e_{\odot}=0$, since 


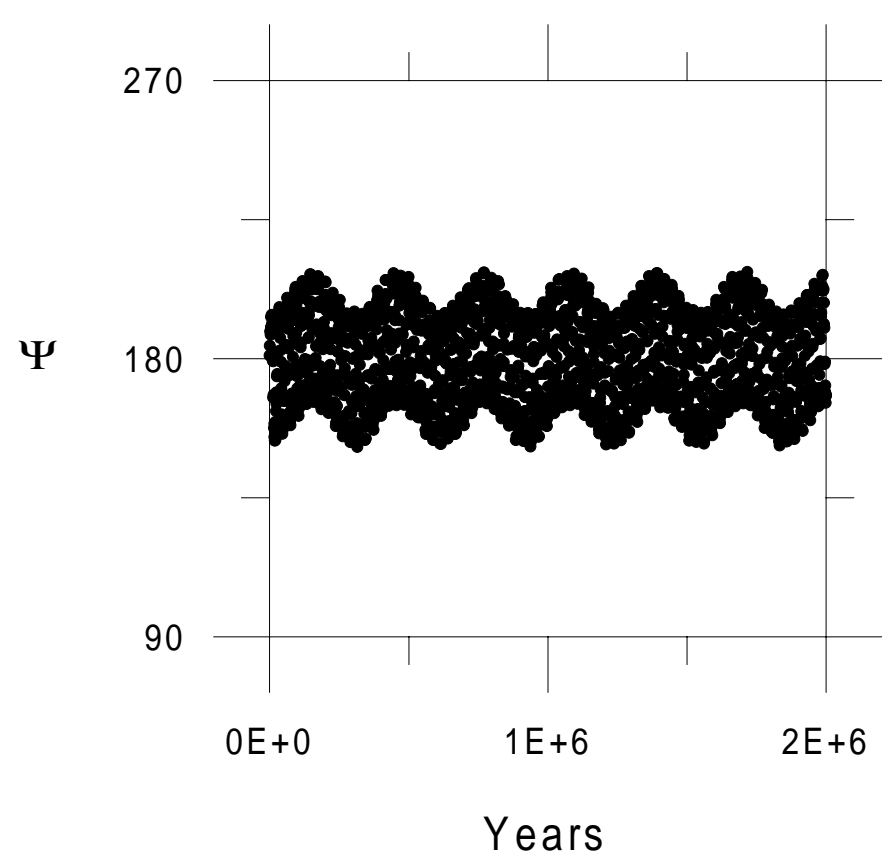

Fig. 3. Direct integration of the satellite-planet-Sun problem confirming the libration shown in Fig. 2B. Here the reference plane is the orbit of the planet, and $\Psi=\varpi-\varpi_{\odot}$.

$\Omega$ disappears in (8). In this case the boundaries of the Kozai resonance (given by the zeroes of the expression inside the curly brackets in Eq. (13)), are related to the solution of:

$$
\begin{aligned}
160 \cos I^{2} & -96+96 e^{2}+\left(-1140 e^{2} \cos I^{2}\right. \\
& +570 e^{4} \cos I^{2}+1470 e^{2} \cos I^{4}-690 \cos I^{2} \\
& \left.+735 \cos I^{4}+270 e^{2}-345 e^{4}+75\right) \rho^{2}=0 .
\end{aligned}
$$

Therefore the inclusion of $\rho^{4}$ terms should not give significant differences mainly for direct satellites, whose $\rho$ are small compared to those of retrograde satellites.

\section{Some numerical integrations}

The role played by the libration of $\varpi-\varpi_{J}$ and some consequences are emphasized both in Whipple \& Shelus (1993) and in Saha \& Tremaine (1993). In the restricted three-body problem, the theoretical possibility of this libration is quite clear, as we demonstrated in the previous section. In this case Jupiter's orbit was fixed. Let us now investigate the behaviour of the resonant angle when $\varpi_{J}$ varies due to the action of extra disturbers, e.g. under the action of the remaining giant planets.

For instance, Fig. 5 refers to the variation with time of $\varpi-\varpi_{J}$ of Pasiphae taking the initial conditions given in Saha \& Tremaine (1993). A very accurate integration based on the Bulirsch-Stoer algorithm (Stoer \& Bulirsch 1980) as given in Mercury's package (Chambers 1999) is used here. The dark and thick curve refers to the actual Pasiphae, while the dotted curve is obtained when its mean anomaly is changed from $l=27.38^{\circ}$ to $l=0^{\circ}$. Figure 5 shows that the period and amplitude of $\varpi-\varpi_{J}$ are quite sensitive to changes in the short-period term. For instance, Whipple \& Shelus (1993) found 13500 years for the libration period of $\varpi-\varpi_{J}$ and $60^{\circ}$
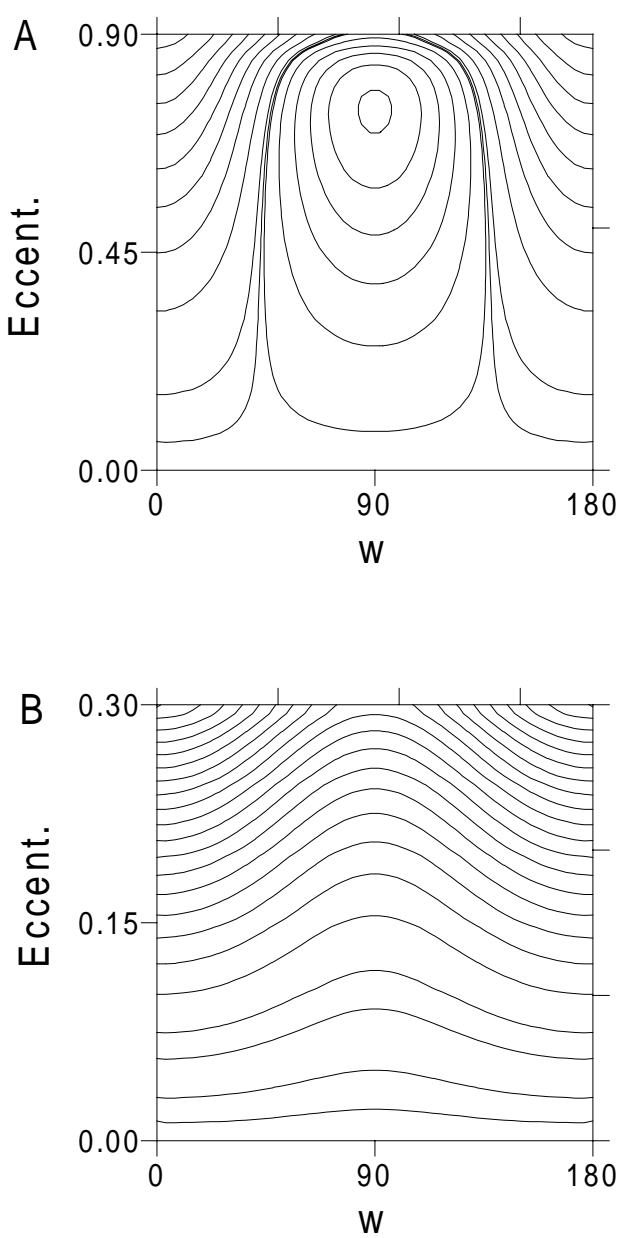

Fig. 4. Kozai resonance: contours for the Hamiltonian (8) for $e_{\odot}=0$. If the inclination falls within the interval $I=\left[39.2^{\circ}, 140.8^{\circ}\right]$, a libration center exist and an initial small eccentricity can attain very high values.

for the amplitude. However due to the sensitivity to initial conditions, definitive values for these quantities probably need accurate observations of the orbits of these satellites. In particular this may be important for Sinope since its $\varpi-\varpi_{J}$ is librating temporally. As before, precise numerical integrations with some slight changes in the initial conditions indicate permanent librations.

Following Huang \& Innannen (1993), and Vieira Neto \& Winter (2001), we will now investigate a large region of the phase space in the neighbourhood of the outer satellites. For the moment, we leave aside the study of chaotic regions or even chaotic diffusion phenomena in the dynamics of ORS. To investigate chaos in dynamical problems there are many sophisticated methods based on the spectral analysis method (Michtchenko 2002, and references therein), frequency analysis (Laskar 1994 and references therein), Lyapunov exponents (Tancredi 2002 and references therein), etc. All of them involve significant computation time. Since in our problem the size of the region we are interested in is very large, we only integrate a grid of initial conditions $(a \times I)$ and plot the maximum eccentricity of the satellite attained during the numerical simulation. This procedure reveals the basic characteristics of the 
dynamics of the motion. We show some regions where the eccentricity remains almost constant (small) while there are other regions where the eccentricity increases rapidly and the satellite is ejected.

In this exploration our time integration is still not long but even so, some interesting regions will appear. Once the location of these particular regions is determined, in a following paper we intend to explore them with much longer integrations.

There is a problem with the choice of the initial conditions used in the numerical simulations. Although it is not clear if the libration of $\varpi-\varpi_{J}$ represents a protection mechanism, in analogy with asteroidal problem, we selected the initial condition satisfying $\varpi-\varpi_{J}=180^{\circ}$ and also:

$\lambda-\lambda_{J}=180^{\circ}$

$\Omega=0^{0}$

$e=0.001$

where for the retrograde orbit, $\varpi=\Omega-w$ and for the planets we used the positions as given in Saha \& Tremaine (1993). We started from almost circular problem because in general capture models assume that capture in libration is certain only if the eccentricity is sufficiently small during the adiabatic evolution of some parameter (e.g. semi major axis or mass variation, see Sect. 6).

Figure 6 shows the numerical integration of a large region around the four outermost satellites. The Mercury package (Chambers 1999) with hybrid option and Radau 15 (Everhart 1985) were used to integrate the orbits for one million years. The step sizes of the grid were: $\Delta a=2$ and $\Delta i=1^{\circ}$. In the lighter region $\left(100^{\circ}<I<140^{\circ}\right)$, the eccentricity attains high values and this is due to the Kozai resonance, as predicted before. Strictly speaking, the four retrograde satellites do not satisfy the initial conditions we used in Fig. 6. However, to obtain a rough view, the averaged positions of Pasiphae, Sinope, Carme and Ananke (see Saha \& Tremaine 1993), are plotted. Even considering horizontal displacements due to variations in the inclination $\left(\delta_{I}= \pm 9.1^{\circ}\right.$ for Pasiphae, $\pm 5.6^{\circ}$ for Sinope, $\pm 3.9^{\circ}$ for Carme, $\pm 5.9^{\circ}$ for Ananke) we see that none of them lie deep inside the critical region of the Kozai resonance.

Around $I \approx 160^{\circ}$ there is an interesting narrow region: a light vertical strip which continues up into the neighbourhood of the ORS. We have not investigated this region in great detail, but some preliminary calculations show that for $a \geq 325$ the angle $\varpi-\varpi_{J}$, and the 6:1 resonance can occur simultaneously. Therefore, as pointed out by Saha \& Tremaine (1993) there is a possibility of weak chaos for this region. A more detailed investigation of the possibility of diffusion along a chaotic route is necessary in the future.

Figure 7 corresponds to the left part of the previous figure, so that the satellites now are prograde (or direct). We have taken values of up to $a=370 R_{J}$ for the semi-major axis; however, due to the computation time, the integration was reduced to 650 thousand years (in most part of the grid) and the step size in the grid for the inclination was set to $\Delta i=2^{\circ}$. The vertical higheccentricity region for $I>50^{\circ}$ is due to the Kozai resonance. In the large high-eccentricity branch starting at $a \approx 310 R_{J}$

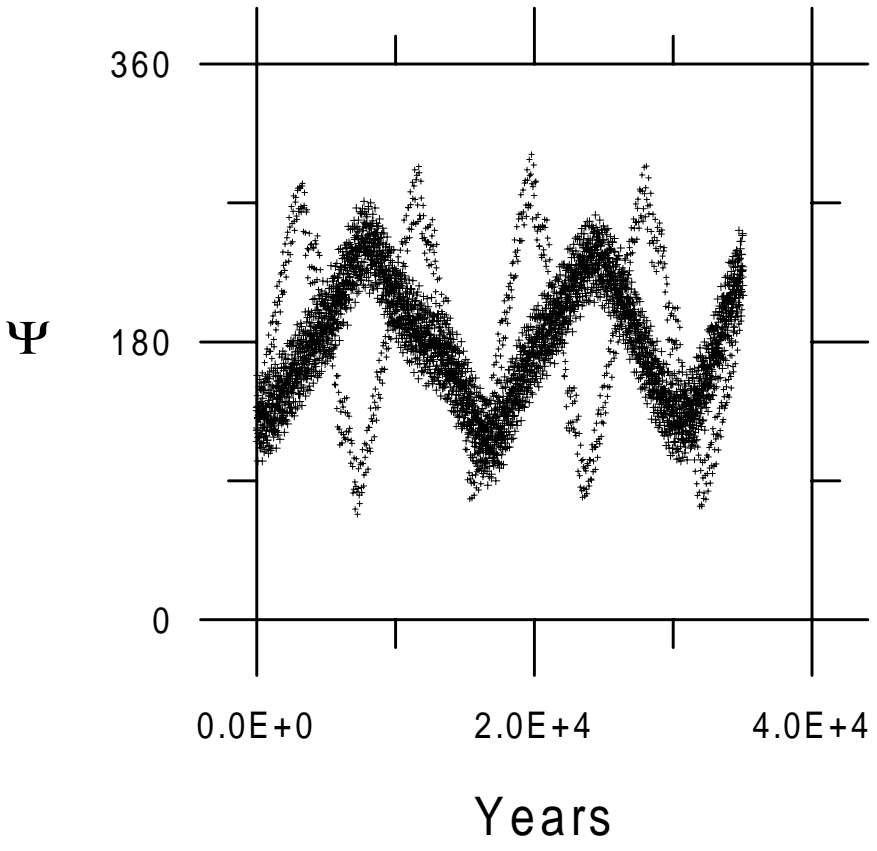

Fig. 5. Variation with time of $\Psi=\varpi-\varpi_{J}$ for Pasiphae, including the effects of the four giant planets. The libration period and the amplitude are very sensitive to slight changes. The only difference in the curves is the initial mean anomaly: $l=27.38^{\circ}$ for the dark (thick) curve and $l=0^{\circ}$ for the dotted curve.

the eccentricity may attain forbidden values. Again, preliminary tests indicate the possible overlapping of different resonances: for $a \approx 324 R_{J}$ we have $\dot{\Omega} \approx 100$ years and a 1:6 orbital resonance between Jupiter and the satellite. Considering $k_{1} \lambda_{S}+k_{2} \lambda_{J}+k_{3} \Omega+k_{4} \varpi_{S}+k_{5} \varpi_{J}$, (where $k_{3}$ is even and $k_{i}$ satisfy D'Alembert's properties), there are several possibilities of additional resonant angles. In particular Fig. 8 shows the time evolution of $\xi=6 \lambda_{J}-\lambda-2 \Omega-3 \varpi_{J}$. Due to the $6: 1$ resonance this angle varies very slowly and when only Jupiter is the disturber $\xi$ librates (Fig. 8A). When all the planets are included the variation of $\xi$ is not well defined, changing from libration to two different modes of circulation.

For $a \approx 292.9 R_{J}$ where another branch starts, we have a 7:1 mean motion resonance and an analogous possibility of overlap.

In Fig. 7 there are some low-eccentricity regions even for $a \approx 300 R_{J}$. For example, the boundaries of the small loweccentricity area (left part), starting at $310 R_{J} \leq a \leq 330 R_{J}$ are well defined, and show some abrupt changes of eccentricity. The integration for this region, i.e., $290 R_{J} \leq a \leq 330 R_{J}$, $2^{\circ} \leq I \leq 43^{\circ}$ was extended to one million years; the general aspects remained unchanged.

If one compares Figs. 7 and 6 it is clear that the area covered with regular orbits is much smaller in the direct case. In Fig. 7 one can see that it would be difficult for a direct satellite to survive in regions where $a \geq 300$, even starting from low inclinations and low eccentricities. In the case of retrograde satellites, the effect of mean motion resonances like 1:6 and 1:7 is very weak and will not increase the initial eccentricity and inclination as it does in Fig. 7. For this reason, direct 
satellites with semi-major axes similar to ORS probably could not survive.

We also considered $\varpi-\varpi_{J}=0$ for the initial condition as given in 15. For Fig. 6 the general aspect is the same while in Fig. 7 the high-eccentricity area for $a \geq 290 R_{J}$ becomes larger than before.

Finally in Fig. 9 we present the integration of a grid around the second group of Jupiter satellites. As in the previous figures, the lighter region for $I>45^{\circ}$ is due to the Kozai resonance. The averaged positions of Himalaia, Lysithea, Elara and Leda are also plotted. Note that all of them are located well inside a low-eccentricity region. The present orbits of these satellites are so clustered that according to Kessler (1981) in the past they could have experienced changes due to collision. For this case we extended some of the integrations up to 10 million years and in principle all orbits remained very similar. In Fig. 9, the only high-eccentricity region is the one caused by the Kozai resonance. The absence of a similar group of satellites in this region suggest that the capture theory gives a natural explanation for the origin of these satellites.

Recently Carruba et al (2002a) showed that even at high inclinations outer satellites with librating $w$ (argument of pericenter) experience a more confined variation in eccentricity than those whose $w$ is circulating. As a consequence these satellites become very stable. For instance, if the stable equilibrium point in Fig. 4 occurs for moderate value of the eccentricity and a particle is trapped in libration, then depending on the amplitude of this libration, the variation of the eccentricity could be confined within secure and moderate limits. Carrying out a numerical integration including the four giant planets, the Sun and other disturbers, Carruba et al. (2002a) demonstrated that satellites trapped in the Kozai resonance (librating $w)$ can be stable for $10 \mathrm{Myr}$ or even more. This result was recently confirmed: at least two Saturnian satellites (S200S5 and $\mathrm{S} / 200 \mathrm{~S} 6$ ) are found to be trapped in this resonance (Carruba et al. 2002b; Cuk et al. 2002) and the integrations showed stability over one Byr. Since in the present paper we have fixed the initial condition $\varpi-\varpi_{J}=180^{\circ}$ (because we are interested in this resonance), our initial $w$ is always $164.72^{\circ}$ (for retrograde orbits) and $195.28^{\circ}$ (for direct orbits), that is, our $w$ is almost always in circulation. On the other hand the quantity $\Theta$ defined in Carruba et al. $(2002 \mathrm{a})$ is this case is: $\Theta \approx \cos ^{2} I$ since our initial $e=0.001$. In this sense, the scenarios presented in Figs. 6 and 7 are not complete because only circulating $w$ cases are considered. This is why in the interval [39.2, 140.8 $]$ we always have very high eccentricity. If $w$ were selected to be in libration, then for small amplitude and convenient constant $H$ (or $\Theta$ ), the general aspect of these figures in the interval $\left[39.2,140.8^{\circ}\right]$ would be changed because some high eccentricity regions (white color) would be replaced by the corresponding low eccentricity regions (black color).

\section{Jupiter's mass growth}

Saha \& Tremaine (1993) point out that it is very curious that Pasiphae is locked in a secular resonance and that Sinope is a temporary librator. They mention that this could be related to the formation of the outer satellites. They suggest
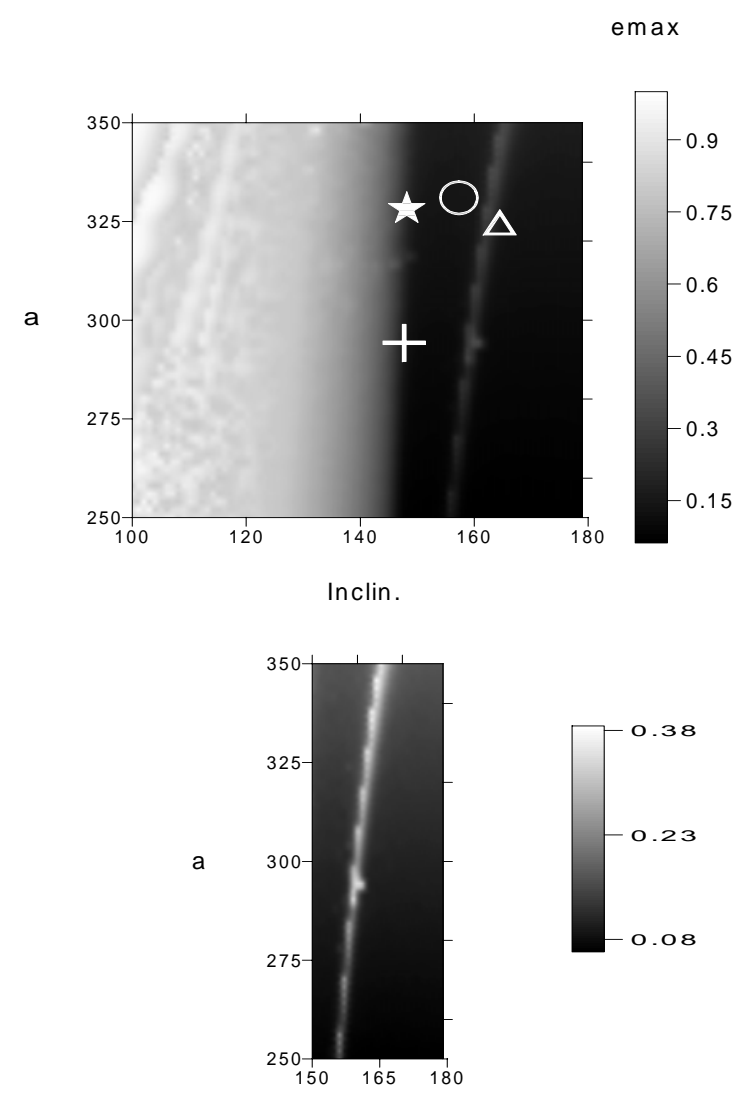

Fig. 6. Numerical integration for the retrograde group of Jupiter's satellites. The values of maximum eccentricity attained during an integration over one million years including the four giant planets are coded in gray levels. As predicted, for retrograde orbits, from $I=90^{\circ}$ to $I \approx 140^{\circ}$, the eccentricity is high due to the action of the Kozai resonance. The lower part shows more details of the complete figure for $150^{\circ} \leq I \leq 180^{\circ}$ : inside a low-eccentricity region, there is a narrow and vertical strip where the eccentricity attains moderate values of up to 0.38 . The meaning of the symbols is: $\Delta=$ Carme, $\star=$ Pasiphae, $\bigcirc=$ Sinope,$+=$ Ananke.

a semi-analytical model where capture into resonances is possible if the orbits of the satellites decay slowly, considering for instance, dissipative effects like mass variation or gas drag. For capture to occur in the Henrard-Lemaître "second fundamental model" (Henrard \& Lemaître 1983), the eccentricity must be very small. Our integrations show that for initial semi-major axes and inclinations similar to those of present retrograde satellites, the eccentricity cannot remain small, even with initial values as low as 0.001 . This means that in order to have small eccentricity, the drag effect must have played an important role (decreasing the eccentricity), otherwise capture would be very difficult. To model these two effects simultaneously causes additional complications in the analytical study. The origin of the high inclination of the ORS and the present libration amplitude of Sinope seem to be additional problems.

In this section we briefly consider the restricted case taking into account only the mass variation of Jupiter. We want to see how the increasing of the mass can influence the possible libration of $\varpi-\varpi_{\odot}$. Neglecting the remaining planets, the numerical problem can be fully integrated if we assume that the mass variation is isotropic. We take a linear growth 


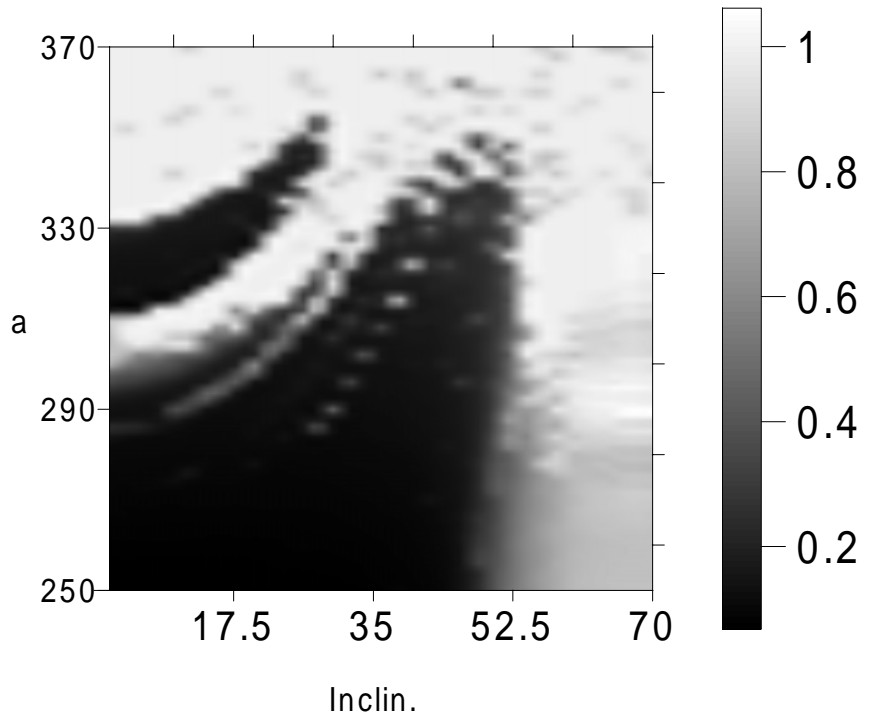

Fig. 7. As in Fig. 6 but for direct satellites. The existence of large regions where the eccentricity is very high does not favor the survival of direct satellites. Because of the Kozai resonance the eccentricity goes to high values in the interval $40^{\circ} \leq I \leq 90^{\circ}$. See text.

$m_{J}=m_{J i}+\beta t$ where $m_{J i}$ is the initial mass and $\beta$ is the mass change scale. Fleming \& Hamilton (2001) consider time scales ranging from $10^{2}$ to $10^{5}$ years, while $m_{J}$ varies from $10 \mathrm{~m} \oplus$ to $320 m \oplus$. At the top of Fig. 10 we present the escape of Carme when Jupiter's mass has decreased from its present value to $85 m \oplus$ in $10^{5}$ years. At about $t=-33770$ years the two-bodyproblem energy becomes positive, so that we assume that escape has taken place. In this case the time scale for $\beta$ is long and before the escape, $\varpi-\varpi_{\odot}$ changes from circulation to libration and back again. Non-autonomous differential equations can be problematic when reversing the time; however, the possibility of a circulation becoming libration and vice versa is clear during the forward integration, (when Jupiter is gaining mass). Figure 10B shows a case of gravitational capture when a forward integration is applied to an escape trajectory. Initially Pasiphae is integrated backwards in time; Jupiter loses its mass, so that Pasiphae escapes. If one reverses the integration, Pasiphae is captured by Jupiter and $\varpi-\varpi_{\odot}$ is also captured in libration. These capture situations do not seem to be so usual; they require some tuning of initial conditions. However, these solutions are possible provided that the differential equations depend continuously on their initial conditions. Additional experiments clearly indicate that mass variation influence the behaviour of $\varpi-\varpi_{\odot}$. To understand these captures into libration further investigations that include gas drag are necessary.

\section{Conclusion}

Although the averaged disturbing function is not efficient when $a / a_{\odot}$ is very large, we assumed that the basic features of secular dynamics are preserved. We have shown analytically the possibility of the libration of $\varpi-\varpi_{J}$ as well as the existence of a new center of libration. The period and amplitude of the librations are quite sensitive to the initial positions. Therefore it seems that precise observations of these satellites are
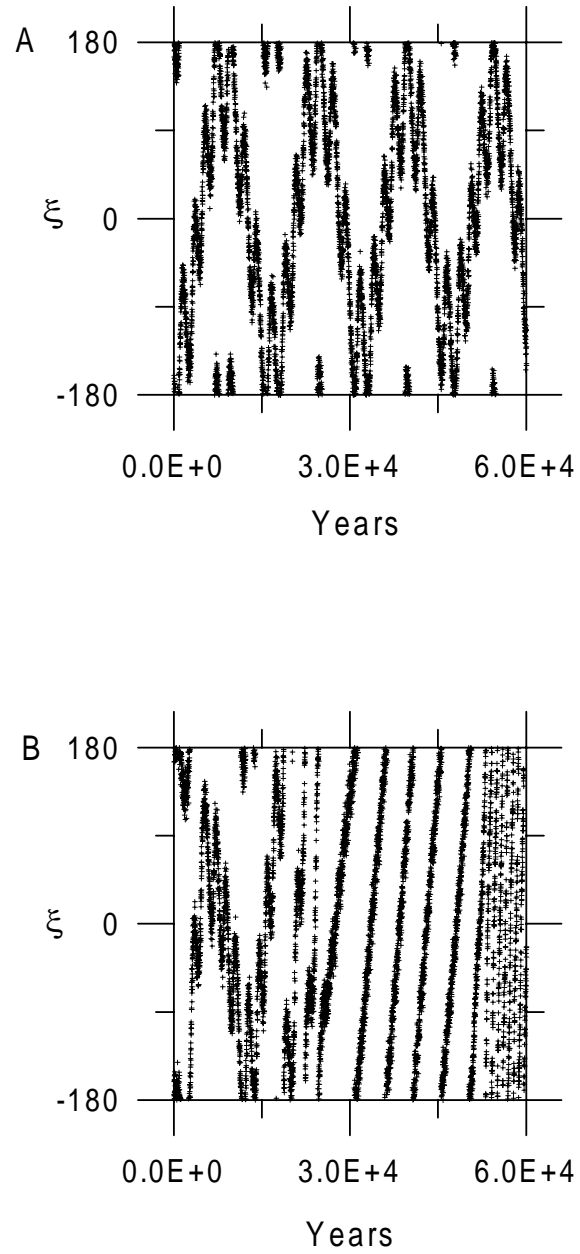

Fig. 8. Time variation of $\xi=6 \lambda_{J}-\lambda-2 \Omega-3 \varpi_{J}$. Initial conditions: $a=326.490, I=15, e=0.001, l=182.14, w=195.28, \Omega=0$. In A (top) where only the Sun is the disturber, $\xi$ librates. In B all other planets are included and the behaviour of $\xi$ changes from libration to two different types of circulation.

necessary to confirm the temporary libration of Sinope. A large grid of initial conditions $(a \times I)$ around the satellites was studied and integrations show the determining role played by the Kozai resonance. It explains that a satellite with circulating $w$ in the interval $40^{\circ} \leq i \leq 141^{\circ}$ undergoes very strong variations in eccentricity so that it can be ejected. For the retrograde satellites there is an interesting vertical strip where eccentricity increases more than one would expect. The direct satellites (Elara, Lisythea, Hymalaia, Leda) are inside a very stable region, and this low-eccentricity area continues up to $a \approx 255 R_{J}$ provided $I \leq 35^{\circ}$, (Fig. 7). However for semi-major axes greater than $290 R_{J}$ and direct objects, there are very large unstable regions. In contrast with retrograde satellites, direct satellites might not not survive at these distances due to the existence of these regions (Fig. 7). We believe that these unstable regions are caused by the overlapping of resonances. More investigations in this direction are in progress. Finally the effect of varying the mass of Jupiter is studied briefly. Some examples are presented showing the possibility of gravitational and libration capture. 


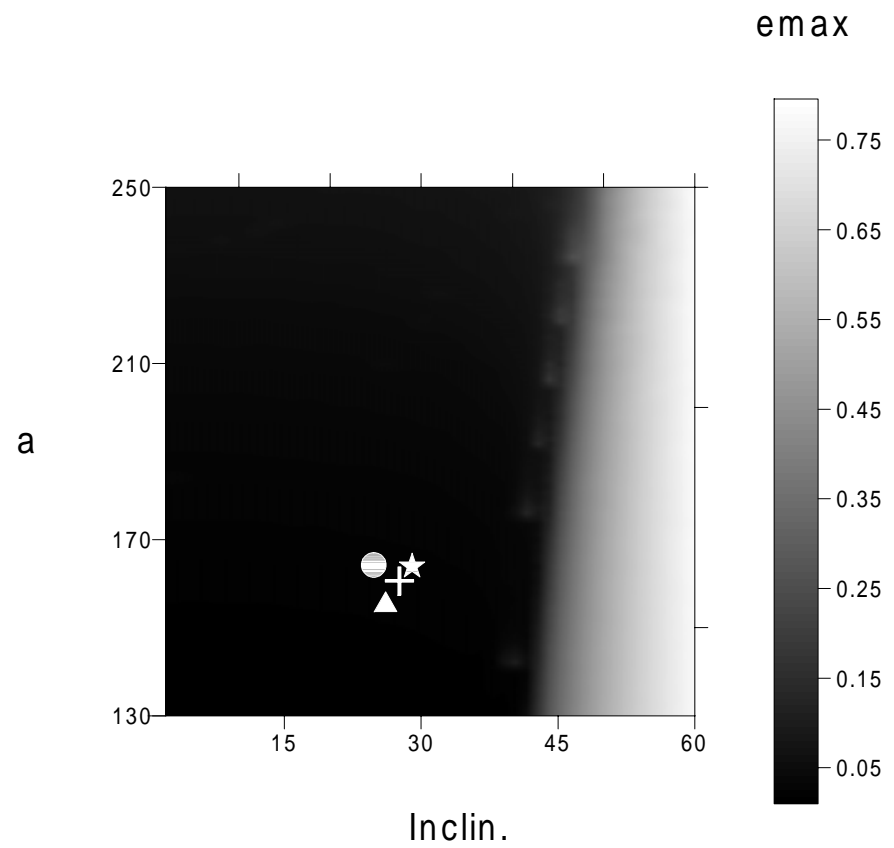

Fig. 9. Results of numerical integration of a grid around the first group of outer satellites. The values of maximum eccentricity attained during an integration over 650 thousand years including the four giant planets are coded in gray levels. As predicted, in the region where the Kozai resonance is effective, the maximum eccentricity is high. $\Delta=$ Leda, $\star=$ Lysithea, $\bigcirc=$ Elara, $+=$ Hymalaia.

Acknowledgements. T. Yokoyama thanks the referee Valerio Carruba for very important suggestions and comments. Part of this work was supported by FAPESP, FUNDUNESP and CNPQ.

\section{References}

Brouwer, D., \& Clemence, G. 1961, Methods of Celestial Mechanics (New York: Academic Press)

Carruba, V., Burns, A. J., Nicholson, P. D., et al. 2002a, Icarus, 158, 434

Carruba, V., Burns, A. J., Nicholson, P. D., et al. 2002b, Bull. Am. Astron. Soc., 34, 902

Chambers, J. E. 1999, MNRAS, 304, 793

Cuk, M., Burns, A. J., Carruba, V., et al. 2002, Am. Astron. Soc., DDA meeting $33,14.02$

Delaunay, C. E. 1860, Mém. Acad. Sci, Paris, 28, 1

Delaunay, C. E. 1867, Mém. Acad. Sci, Paris, 29, 1

Everhart, E. 1985, in Dynamics of Comets: Their Origin and Evolution, ed. A. Carusi, \& G. Valsecchi (Dordrecht: Reidel), 185

Fleming, H. J., \& Hamilton, D. P. 2000, Icarus, 148, 479

Gladman, B. J., Nicholson, P. D., Burns, J. A., et al. 1998, Nature, 392, 1998

Gladman, B. J., Kavelaars, J. J., Holman, M., et al. 2000, Icarus, 147, 320

Gladman, B. J., Kavelaars, J. J., Holman, M., et al. 2001, Nature, 412, 163

Henrard, J., \& Lemaître, A. 1983, Celest. Mech. Dyn. Astr., 30, 197

Hénon, M. 1965a, Ann. Astr., 28, 499

Hénon, M. 1965b, Ann. Astr., 28, 992
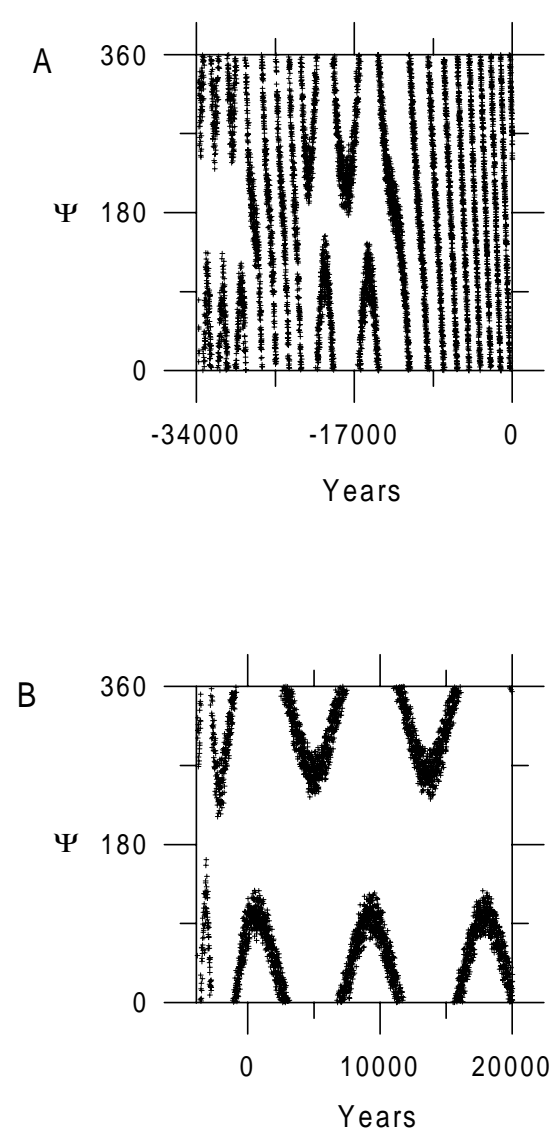

Fig. 10. Top: the orbit of Carme is integrated with time reversed, decreasing the mass of Jupiter. The change from circulation-librationcirculation of $\varpi-\varpi_{\odot}$ is due to the variation of the mass. Bottom: after an escape, reversing the decrease of mass, Pasiphae is captured by Jupiter and also in $\varpi-\varpi_{\odot}$ libration. The situation remains unchanged when Jupiter's mass is stabilized.

Hénon, M. 1966a, Bull. Astr. Paris, 1, 1, 57

Hénon, M. 1966b, Bull. Astr. Paris, 1, 2, 49

Hénon, M. 1969, A\&A, 1, 223

Hénon, M. 1970a, A\&A, 9, 24

Hénon, M., \& Guyot, M. 1970b, in Periodic Orbits, Stability and Resonances, ed. G. E. O. Giacaglia (Dordrecht: Reidel), 349

Huang, T. Y., \& Innanen, K. A. 1983, AJ, 88, 1537

Kessler, D. 1981, Icarus, 48, 39

Laskar, J. 1988, A\&A, 198, 341

Laskar, J. 1994, A\&A, 287, L9

Michtchenko, A. T., Lazzaro, D., Ferraz-Mello, S., et al. 2002, Icarus, 158,343

Moons, M. 1994, Celest. Mech. \& Dyn. Astr., 60, 173

Nascimento, C., \& Yokoyama, T. 2003, in preparation

Sheppard, S. S., \& Jewit, D. C. 2002, Bull. Am. Astron. Soc., 34, 881

Tancredi, G., Sánches, A., \& Roig, F. 2002, AJ, 121, 1171

Saha, P., \& Tremaine, S. 1993, Icarus, 106, 549

Stoer, J., \& Bulirsch, R. 1980, Introduction to Numerical Analysis (New York: Springer-Verlag)

Vieira Neto, E., \& Winter, O. C. 2001, AJ, 122, 440

Whipple, A., \& Shelus P. J. 1993, Icarus, 101, 265 